\title{
O típico do cuidado de enfermagem ao prematuro em relação ao sono e a vigília
}

\section{The typical caring action of nursing in relation to sleep-wake cycle of preterm infant}

\author{
Suzana Quintiliano da Silva ${ }^{1}$, Myriam Aparecida Mandetta ${ }^{2}$, Maria Magda Ferreira Gomes Balieiro ${ }^{3}$ \\ ${ }^{1}$ Enfermeira. Enfermeira da Unidade de Pronto Atendimento Ibirapuera do Hospital Israelita Albert Einstein. São Paulo, SP, Brasil. E-mail: suzana_qs@ @ hotmail.com. \\ ${ }^{2}$ Enfermeira, Doutora em Enfermagem. Professor Associado da Escola Paulista de Enfermagem (EPE) da Universidade Federal de São Paulo (UNIFESP). São Paulo, SP, \\ Brasil. E-mail: mpettengill@unifesp.br. \\ ${ }^{3}$ Enfermeira, Doutora em Enfermagem. Professor Associado da EPE/UNIFESP. São Paulo, SP, Brasil. E-mail: mmfgbalieiro@unifesp.br.
}

\section{RESUMO}

Pesquisa qualitativa fenomenológica realizada com o objetivo de compreender o típico da ação de cuidar dos técnicos de enfermagem em relação ao sono e vigília de recém-nascidos prematuros internados em uma unidade neonatal. Participaram nove sujeitos vinculados a um hospital público do município de São Paulo, por meio de entrevistas semiestruturadas. A categoria "agir para preservar o bem-estar do prematuro" está ancorada em conhecimentos, experiências e crenças prévias dos profissionais em relação à interpretação dos sinais emitidos pelo prematuro; a importância do sono; e os efeitos dos estímulos ambientais. Esta ação é direcionada pelo desejo de realizar assistência com qualidade, garantindo alta hospitalar mais precoce e sem sequelas. Conclui-se que o agir dos técnicos de enfermagem vai ao encontro do modelo do cuidado desenvolvimental do prematuro. Recomenda-se a inclusão da temática sobre a individualização do cuidado ao prematuro e de sua família na formação dos técnicos de enfermagem. Descritores: Sono; Vigília; Prematuro; Enfermagem Neonatal; Unidades de Terapia Intensiva Neonatal.

\section{ABSTRACT}

A qualitative study, grounded on social phenomenology of Alfred Schütz was conducted to understand the typical caring action of nursing technicians regarding the sleep-awake cycle of premature babies admitted at a neonatal unit. Nine members of a nursing staff from a public hospital of São Paulo participated through semi-structured interviews. Through the data analysis emerged the category "acting to preserve the wellbeing of premature babies", based on the staff knowledge, experiences and previous beliefs about the interpretation of signs from the babies, on the importance of sleep, and on the effects of environmental stimuli over this human being. These actions are directed with the intention to improve the quality of nursing care for babies at neonatal unit with the aim to promote their early hospital discharge without any sequel. We concluded that actions from nursing technicians meet the neonatal developmental care model. We recommend the thematic inclusion of the neonatal care individualization and its family in the training of nursing technicians.

Descriptors: Sleep; Wakefulness; Neonatal Nursing; Intensive Care Units, Neonatal. 


\section{INTRODUÇÃO}

A aplicação do modelo do cuidado desenvolvimental para assistir ao prematuro desde seu nascimento tem sido relacionada com o impacto na redução da dor e do estresse sobre seu cérebro ${ }^{(1-7)}$, a melhora da estabilidade clínica, favorecendo o ganho ponderal, a promoção da organização comportamental, com diminuição do tempo de alimentação enteral e de complicações hemorrágicas, além de promoção da adaptação familiar ${ }^{(8)}$.

Este modelo de cuidado preconiza a alteração do ambiente de cuidado, a implementação de medidas para promover a autorregulação, o manuseio e o posicionamento adequados do $\mathrm{RN}$, o planejamento conjunto dos cuidados, a amamentação, a preservação do sono, do contato pele-a-pele, o envolvimento dos pais nos cuidados do seu filho e uma relação individualizada com o recém-nascido, indicando assim o respeito para com este $\operatorname{ser}^{(3-5,8-11)}$.

Um estudo sobre os efeitos do sono no desenvolvimento emocional e social da criança identificou que o sono durante o dia pode proporcionar uma oportunidade para o prematuro "reagrupar-se" ou "reorganizar-se", com efeitos sobre a aprendizagem e a regulação do cortisol; além de efeitos sobre a qualidade da parentalidade e da ligação mãe-bebê $\hat{~}^{(10,12)}$.

Em uma revisão sistemática ${ }^{(11)}$ incluindo 627 prematuros, os autores não encontraram nenhuma evidência que o cuidado desenvolvimental melhora os resultados clínicos dos $\mathrm{RNs}$ e nem o desenvolvimento neurológico a curto ou longo prazo. Porém, os autores referem que o foco central desse modelo de cuidado é a interação humana. Assim, o cuidado desenvolvimental é considerado padrão de melhores práticas no cuidado do prematuro nas unidades neonatais ${ }^{(8)}$.

Todos os profissionais da equipe de enfermagem tem o papel de organizar o ambiente da unidade neonatal, sob orientação do enfermeiro, que deve planejar a assistência de maneira individualizada, facilitando a interação entre pais e filhos pois a prática do cuidado desenvolvimental requer envolvimento da equipe e dos pais para a identificação dos sinais comportamentais do prematuro $^{(8)}$.

No Brasil, considerando-se que os técnicos de enfermagem são o maior contingente da equipe de enfermagem na execução de ações de cuidado ao prematuro, questiona-se como esses profissionais incorporam as premissas do cuidado desenvolvimental em sua prática e o que os mobiliza nesse sentido.

Assim, é fundamental a compreensão da prática dos técnicos de enfermagem na adoção dos princípios do cuidado desenvolvimental, buscando caracterizar suas ações de cuidado, os motivos que as embasam e os que os mobilizam para a realização de uma prática que contemple o cuidado com o sono e vigília do prematuro.

O objetivo deste estudo foi compreender o típico da ação de cuidar dos técnicos de enfermagem em relação ao sono e vigília de recém-nascidos prematuros internados em uma unidade neonatal.

\section{MÉTODO}

Estudo de abordagem qualitativa, fundamentado na fenomenologia social de Alfred Schütz, definida como "o estudo do modo como as pessoas vivenciam o cotidiano $e$ imbuem de significado as suas atividades"(13).

O estudo foi realizado na unidade neonatal de um hospital estadual de São Paulo, composta de 12 leitos distribuídos em berçário de médio risco I e II, sala de cuidados semi-intensivos e 10 leitos de unidade de terapia intensiva neonatal. A equipe de enfermagem é constituída por um enfermeiro supervisor, um enfermeiro assistencial e nove técnicos de enfermagem em cada turno de trabalho.

Na unidade, existem protocolos para a prevenção do ruído ambiental e redução de luminosidade. Diariamente, são programados quatro momentos de descansos denominados "horários de soninho", com duração de uma hora cada, com redução das luzes do ambiente, cessação de manipulação do RN e diminuição do timbre de voz dos profissionais. 
Participaram nove técnicos de enfermagem que atendiam aos critérios de inclusão: ser colaborador da unidade neonatal e estar desenvolvendo suas atividades nas salas de cuidados intermediários; e aos critérios de exclusão: ser colaborador em período de férias ou afastamentos e em cobertura de folgas. A delimitação do número de participantes foi definida no transcorrer da coleta de dados baseada nas convergências dos discursos e na compreensão do fenômeno.

A coleta de dados foi realizada, no ano de 2010, por meio de entrevista semiestruturada, gravada e focalizada na questão norteadora: Como é o seu cuidado ao recémnascido prematuro quanto ao sono e a vigília na unidade neonatal? O que você visa ao realizar esses cuidados?

Para análise dos depoimentos, utilizaram-se as etapas recomendadas por pesquisadores ${ }^{(14)} \mathrm{da}$ fenomenologia de Alfred Shütz procurando-se focar, de forma atenta, naquilo que se apresentava em comum, isto é, buscando os "motivos por que" e os "motivos para" revelados nos discursos e identificados em unidades de significados, que formaram as categorias concretas emergidas do vivido, permitindo ao final a descrição do tipo vivido dos técnicos da equipe de enfermagem.

O desenvolvimento do estudo atendeu as normas nacionais e internacionais de ética em pesquisa envolvendo seres humanos, sendo submetido ao Comitê de Ética em Pesquisa da instituição de vinculação dos pesquisadores e aprovado pelo protocolo CEP 1745/09.

\section{RESULTADOS}

Os técnicos de enfermagem buscam agir para preservar o bem-estar do prematuro na unidade neonatal, por meio da execução de cuidado ao sono e vigília, sustentados nos conhecimentos que possuem (motivo por que) das respostas fisiológicas e comportamentais do neonato, da crença sobre a importância do sono para o prematuro, dos efeitos dos estímulos ambientais e da rotina estabelecida na unidade neonatal. A ação do profissional caracteriza-se pela manipulação cuidadosa do prematuro; redução dos estímulos ambientais na unidade neonatal; atendimento às necessidades básicas do prematuro; comunicação com o prematuro; promoção da organização do prematuro; preservação do sono e manutenção do ritmo circadiano do prematuro.

As motivações profissionais para o cuidado do prematuro têm como expectativas (motivos para que), a prestação de assistência de enfermagem de qualidade para este cliente, a fim de reduzir o tempo de internação e proporcionar alta hospitalar sem sequelas.

\section{Motivos por que}

Neste estudo as razões do agir dos técnicos de enfermagem estão baseadas no conhecimento teórico e nas experiências pessoais e profissionais quanto à importância do sono para o prematuro.

Os profissionais reconhecem que o sono promove o crescimento e o desenvolvimento; a reposição de energia; tranquilidade; redução de estresse; a recuperação da saúde e a estabilização do quadro clínico do prematuro. Referem ainda que preservar o sono do prematuro é um cuidado que o organiza, pois ele fica mais tranquilo, protegido, com a estabilização de suas funções vitais e ganho ponderal diário. O sono é fundamental, tanto para estabilização, quanto para ganho de peso do bebê (E8).

Os técnicos de enfermagem usam a interpretação das respostas comportamentais do prematuro aos estímulos ambientais, ao toque e à manipulação como forma de reconhecimento de sua condição. A decodificação dos sinais emitidos pelo bebê tem o intuito de proporcionar uma melhor organização do neonato e um período de sono que favoreça sua estabilização clínica.

Na manipulação, só de você abrir a incubadora e tocar (bebê), alguns até empurram a mão, eles empurram a mão da gente, quer dizer que está incomodando. Quando você coloca-o em uma posição que gosta, posição ventral, por exemplo, que eles gostam muito e deixa quietinho, 
eles relaxam e dormem, você vê que a feição está bem relaxadinha (E2).

Outro aspecto que sustenta as ações dos profissionais é o conhecimento dos efeitos dos estímulos ambientais (som, luz e manipulação frequente) para o sono da criança. Para os profissionais, a unidade neonatal é um ambiente que precisa manter uma rotina de maior controle quanto às condições clínicas do $\mathrm{RN}$, impondo a presença de vários equipamentos de monitorização e maior número de profissionais de saúde, que dificultam o reconhecimento das pistas comportamentais e fisiológicas emitidas pelo recém-nascido. A instabilidade da criança leva a manipulações que associadas a um ambiente ruidoso determinam ao prematuro um período de sono curto. O excesso de manipulação do prematuro, o som dos alarmes e das vozes dos profissionais dificultam sua permanência em sono regular ou quieto.

O conhecimento desses aspectos e a resposta comportamental da criança a esses estímulos são as razões, que levam os profissionais de enfermagem a elegerem suas intervenções quanto ao sono e vigília.

Há um movimento muito grande tanto dentro da UTI como no berçário, e a voz, os aparelhos, alarmes [...] É uma coisa que infelizmente a gente não tem muito sucesso aqui dentro do berçário, não... (E8).

Estas ações caracterizam-se pela manipulação mínima e cuidadosa, redução dos estímulos ambientais, atendimento das necessidades básicas do prematuro, comunicação, promoção da organização do prematuro, preservação do sono e manutenção de um ritmo.

A manipulação mínima foi revelada como a concentração das atividades da equipe de saúde da unidade, para que centralize os cuidados prestados ao prematuro, realizando várias ações ao mesmo tempo e com menor frequência.
A gente evita ao máximo estar manipulando os bebês, porque tudo que a gente vai fazer a gente procura fazer tudo de uma vez, controle dos sinais vitais, se tem medicação de horário a gente já faz, porque quanto menos a gente manipula o bebê melhor é pra ele, para o soninho, porque ele acaba descansando melhor... (E6).

Outra forma de agir é a abordagem gradativa do neonato, que se caracteriza pelo despertar do bebê antes dos procedimentos, de maneira cautelosa e gradual, evitando que ele se assuste e fique estressado.

O atendimento das necessidades básicas do prematuro está presente nos discursos dos técnicos de enfermagem, como um fator determinante para que o prematuro possa ter um sono tranquilo e duradouro na unidade neonatal. Os profissionais procuram manter o conforto, por meio de mudança constante de decúbito, aquecimento, ambiente calmo e organização, com o uso de rolos e de coxins. Quando essas necessidades são atendidas, a equipe percebe que o RN dorme com tranquilidade e acorda apenas para alimentar-se ou na presença de dor.

A comunicação com o prematuro é entendida como uma abordagem que respeita sua prontidão para a interação com o outro. As estratégias descritas para a comunicação são conversar com o RN antes e durante os procedimentos ou manipulações, explicando-lhe o que será feito pelo profissional como forma de evitar comportamentos de afastamento. Os técnicos de enfermagem reconhecem os sinais de estresse ou de afastamento emitidos pelos prematuros, e promovem sua organização, por intermédio de estratégias de contingenciamento ou por redução de manipulação.

Os profissionais percebem que quando realizam um procedimento invasivo sem a avaliação da prontidão da criança para a interação, geram intensificação de seu choro, irritação e estresse. Desta forma, acreditam que quando reduzem o toque para o mínimo possível e preservam o horário de sono do $\mathrm{RN}$ promovem seu descanso tornando-o mais organizado. 
Outra categoria que aponta a ação social deste grupo para promover o bem-estar é a manutenção de um ritmo para o prematuro, definido como a determinação de horários na realização dos cuidados para que ele se adapte às rotinas da unidade neonatal.

\section{Motivos para}

Neste estudo, os motivos para, são a promoção de assistência de enfermagem de qualidade, com a alta hospitalar precoce do $\mathrm{RN}$ sem sequelas.

No discurso dos técnicos de enfermagem evidenciase que há uma busca por proporcionar um cuidado efetivo e de qualidade ao prematuro, trazendo-lhe tranquilidade e tratando-o de forma afetiva e com empatia.

Os profissionais têm a necessidade frequente de aperfeiçoamento, para que mantenham a qualidade do atendimento ao RN e assim promover um cuidado seguro.

Eu acho que tem sempre que estar se aperfeiçoando mais, indo à busca de mais para dar ao bebê o maior conforto e proteção possível (E4).

Ao perceber que a assistência está sendo benéfica ao $\mathrm{RN}$, trazendo-Ihe a recuperação da saúde, os profissionais sentem-se satisfeitos e realizados. A gratificação é intensificada quando avaliam na consulta de retorno que a criança cresceu e está saudável.

Porque é gratificante você encontrar uma mãe, que veio para uma consulta e ver que o bebê está bem, grande, você sabe que você deu o seu melhor e que aquilo surtiu efeito (E9).

Os técnicos de enfermagem referem que cuidam do prematuro com amor, para que ele possa ganhar peso rapidamente e receber alta hospitalar em boas condições sem grau de dependência futura.

\section{DISCUSSÃO}

Ação social é "uma conduta entre duas ou mais pessoas, projetada pelo ator de maneira consciente"; que é intencional e tem um significado subjetivo que direciona o ator, que pode se orientar para o passado, presente ou futuro $^{(13)}$.

Neste estudo, a ação social da equipe de enfermagem é a preservação do bem-estar do prematuro. Esta ação é influenciada por seus conhecimentos e vivências passadas, direcionadas à redução das repercussões da unidade neonatal na saúde do prematuro. Muitas das ações desveladas nos discursos dos profissionais baseiam-se nos pressupostos do cuidado desenvolvimental.

Este modelo de cuidado refere-se a combinações de filosofias e técnicas de intervenção focadas na criança, como a figura central na unidade neonatal, promovendo seu conforto, crescimento e desenvolvimento, sempre respeitando suas individualidades ${ }^{(15)}$.

No entanto, ainda se observa no discurso desses profissionais que apesar da preocupação com a individualidade do prematuro, nem sempre é possível garanti-la, uma vez que a meta de suas ações é a manutenção de um ritmo para o prematuro. Daí empreender uma rotina de horários rígidos para alimentação, sono, higiene e troca de fraldas que dificulta a relação face a face, como o preconizado pelo modelo do cuidado desenvolvimental.

Schütz refere que "meu semelhante é sempre alguém particular, não outro qualquer, mas alguém que para mim tem um nome e particularidades" ${ }^{(13)}$. Neste estudo, o discurso dos técnicos de enfermagem revela o compromisso com o cuidado face a face, de respeito e decodificação dos sinais emitidos pelo prematuro. Quando os técnicos de enfermagem interagem pela relação face a face, em um modo de aproximar-se e interagir com o prematuro, eles são capazes de interpretar os estados comportamentais do RN antes, durante e após uma manipulação e identificar sinais de não adaptação ao ambiente extrauterino. Este cuidado 
individualizado é referido pelos autores como uma intervenção realizada no momento adequado e de forma a respeitar a prontidão do neonato ${ }^{(15)}$.

A humanização da assistência na unidade neonatal não consiste apenas em prestar cuidados ao prematuro, mas também é de grande importância que haja um acolhimento de sua família, incluindo-a nos cuidados ao bebê, a fim de facilitar o vínculo e o apego precocemente, preparando os pais para o cuidado domiciliar do RN após a alta. Entretanto, a família neste estudo, ainda, não é considerada pelos técnicos de enfermagem, como um apoio para preservar o bem-estar do prematuro quanto ao sono e vigília.

A inserção dos pais nos cuidados ao $\mathrm{RN}$ faz parte das estratégias do cuidado desenvolvimental, pois favorece o desenvolvimento neurológico da criança e facilita a ligação afetiva entre eles, além de servir de aprendizado para os pais cuidarem do filho em casa, trazendo-lhes mais segurança ${ }^{(16)}$.

Neste estudo, os profissionais apontaram a manipulação mínima e cuidadosa, com diminuição dos estímulos ambientais, como ruído e luminosidade como importantes para a redução do desconforto provocado ao neonato na unidade neonatal e a promoção de um período maior de sono. Outro aspecto desvelado foi a comunicação dos profissionais com o RN antes e durante as manipulações, para mantê-lo organizado.

Apesar dos benefícios do cuidado desenvolvimental demonstrados em diversos estudos ${ }^{(1,3-4,7-8,17)}$ uma revisão sistemática recentemente publicada sobre a temática ${ }^{(11)}$, afirma que esta prática não traz evidências quanto a melhora dos resultados neuro-desenvolvimentais a curto e longo prazo. Aspecto, que necessita de estudos clínicos sobre a efetividade deste cuidado, com avaliação isolada de cada ação proposta por este modelo.

Vale ressaltar que os aspectos de preservação do bem-estar do prematuro levantados neste estudo são referentes ao discurso dos técnicos de enfermagem, porém permanece o questionamento sobre como se mostra o comportamento dos enfermeiros e equipe de enfermagem no cotidiano da unidade neonatal.

O uso do modelo do cuidado desenvolvimental por enfermeiros iranianos, em uma unidade de terapia intensiva neonatal, foi avaliado quanto ao cuidado individualizado, ambiente e apoio ao relacionamento pais-crianças. Os resultados apontaram que $76 \%$ das ações dos enfermeiros são congruentes às premissas desse modelo de cuidado. Assim, recomendam a capacitação de toda a equipe de saúde sobre este aspecto e uma menor relação número de pacientes e enfermeiro. Além, da adoção de medidas estruturais e funcionais para a efetivação do cuidado desenvolvimental ${ }^{(18)}$.

Em outra pesquisa sobre as barreiras relatadas por enfermeiros para a realização do cuidado desenvolvimental foi identificado a falta de conhecimento do método, limitação do horário da família na unidade neonatal, falta de habilidade de comunicação para discutir estas questões com os pais, falta de tempo devido à carga de trabalho e pouca autonomia na prestação do cuidado neonatal $^{(19)}$.

Na literatura internacional não há estudos enfocando a prática do cuidado desenvolvimental envolvendo técnicos de enfermagem, por ser uma característica da divisão do trabalho de enfermagem no Brasil. No entanto, na literatura nacional, também não foi encontrado nenhuma pesquisa com esses sujeitos. Pode-se fazer uma suposição sobre as barreiras para a implementação desse modelo de cuidar na perspectiva dos técnicos de enfermagem, a partir dos resultados de pesquisas com enfermeiros, considerando que estes estão hierarquicamente sob supervisão do enfermeiro.

A presente pesquisa, por ser um estudo qualitativo, sobre o discurso dos técnicos de enfermagem a respeito do cuidado ao prematuro em relação ao sono e vigília, descortinou uma faceta desse fenômeno, mantendo ainda oculto como ocorre esse cuidado na ação cotidiana da equipe de saúde, revelando as barreiras para a sua implementação e as evidências dos benefícios deste cuidado por meio de estudos clínicos. 


\section{CONCLUSÃO}

Os técnicos de enfermagem agem para preservar o bem-estar do prematuro na unidade neonatal, por meio da execução de cuidado ao sono e vigília, sustentados nos conhecimentos das respostas fisiológicas e comportamentais do neonato, da crença sobre a importância do sono para o prematuro, dos efeitos dos estímulos ambientais e da rotina estabelecida na unidade neonatal.

As motivações profissionais para o cuidado do prematuro têm como expectativas a prestação de

\section{REFERÊNCIAS}

1. Als H, Duffy FH, McAnulty G, et al. NIDCAP improves brain function and structure in preterm infants with severe intrauterine growth restriction. J Perinatol. 2012;32:797-803

2. Grecco GM; Tsunemi MH; Balieiro MMFG; Kakehashi TY, Pinheiro EM. Repercussion of noise in the neonatal intensive care unit Acta paul. enferm. 2013; 26(1):1-7.

3. McAnulty G1, Duffy FH, Kosta S, Weisenfeld NI, Warfield SK, Butler SC, Alidoost M, Bernstein JH, Robertson R, Zurakowski D, Als H. Schoolage effects of the newborn individualized developmental care and assessment program for preterm infants with intrauterine growth restriction: preliminary findings. BMC Pediatr. 2013;13:25. doi: 10.1186/1471-2431-13-25

4. Mosqueda R, Castilla Y, Perapoch J, Cruz J, Lòpez-Maestro M, Pallàs C. Staff perceptions on newborn individualized developmental care and assessment program (NIDCAP) during its implementation in two Spanish neonatal units. Early Human Development 2013; 89:27-33 5. Lins RNP, Collet N, Vaz EMC, Reichert AP. Percepção da equipe de enfermagem acerca da humanização do cuidado na UTI Neonatal. Revista Brasileira de Ciências da Saúde 2013; 17(3):225-232. 6. Santos BR; Orsi KCSC; Balieiro MMFG; Sato MH; Kakehashi TY; Pinheiro EM. Effect of "quiet time" to reduce noise at the neonatal intensive care unit. Esc. Anna Nery 2015;19(1):102-106.

7. Lawhon G, Helm JM, Buehler D, McAnulty G, Kosta S, Alberts JR, Als H, Mader S, Daly M, Sizun J, Vandenberg K, Warren I. NIDCAP Federation International response. Pediatrics 2013;132(2):e550-1. 8. Gibbins S, Hoath SB, Coughlin M, Gibbins A,Franck L. The universe of developmental care: a new conceptual model for application in the neonatal intensive care unit. Adv Neonatal Care 2008;8(3):141-147 9. Gaiva MAM, Marquesi MC, Rosa MKO. O sono do recém-nascido internado em unidade de terapia intensiva: cuidados de enfermagem. Cienc. Cuid. Saúde. 2010; 9(3):602-09.

10. Shah PE, Poehlmann J. Sleep and Attachment in Preterm Infants. Infant Ment Health J. 2013; 34(1): 37-46.

11. Ohlsson A, Jacobs SE. NIDCAP: a systematic review and metaanalyses of randomized controlled trials. Pediatrics 2013; 31(3):e88193

12. Schwichtenberg AJ, Shah PE, Poehlmann. Sleep and Attachment in Preterm Infants. J. Infant Ment Health J. 2013; 34(1): 37-46.

13. Schütz A. El problema de la realidad social: escritos I. 2ª ed. Buenos Aires: Amorrortu, 2003. assistência de enfermagem de qualidade para este cliente, a fim de reduzir o tempo de internação e proporcionar alta hospitalar sem sequelas.

$\mathrm{O}$ agir dos técnicos de enfermagem para preservar o bem-estar do prematuro vai ao encontro do modelo de cuidado desenvolvimental, é movido por uma relação face a face com o RN, apesar de algumas vezes ser realizado de forma rotineira e a família não estar incluída nesse contexto.

14. Conz CA, Merighi MAB, Jesus MCP. Promoting affective attachment at the neonatal intensive care unit: a challenge for nurses. Rev. esc. enferm. USP 2009; 43(4): 849-55

15. Als H, Duffy FH, McAnulty GB, Fischer CB, Kosta S, Butler SC, Parad RB, Blickman JG, Zurakowski D, Ringer SA. Is the Newborn Individualized Developmental Care and Assessment Program (NIDCAP) effective for preterm infants with intrauterine growth restriction? J. Perinatol. 2011;31(2):130 -136.

16. Gooding JS, Cooper LG, Blaine AI, Franck LS, Howse JL, Berns SD. Family Support and Family-Centered Care in the Neonatal Intensive Care Unit: Origins, Advances, Impact. Semin Perinatol 2011; 35(1):2028

17. McAnulty GB, Butler SC, Bernstein JH, Als H, Duffy FH, Zurakowski D. Effects of the Newborn Individualized Developmental Care and Assessment Program (NIDCAP) at Age 8 Years: Preliminary Data. Clinical Pediatrics 2010; 49(3);259-270.

18. Valizadeh L, Asadollahi M, Gharebaghi MM, Gholami M. The Congruence of Nurses' Performance with Developmental Care Standards in Neonatal Intensive Care Units. Journal of Caring Sciences 2013;2(1),61-71.

19. Zhang X, Lee SY, Chen J, Liu H. Factors Influencing Implementation of Developmental Care Among NICU Nurses in China. Clin Nurs Res [internet] 2014;1-16. Available from:

http://www.ncbi.nlm.nih.gov/pubmed/25155801.

Recebido: 20/03/2014.

Aceito: 18/03/2015.

Publicado: 30/06/2015. 